\title{
Investigaciones sobre los efectos de la neoliberalización de la educación superior pública en América Latina'
}

\author{
Isaura Castelao-Huerta² \\ http://orcid.org/0000-0001-9402-3868
}

\section{Resumen}

El presente artículo es una revisión de la literatura publicada entre 1995 y 2019 acerca de los efectos de la aplicación de políticas neoliberales en la educación superior pública en América Latina. Da cuenta de cómo el recorte al presupuesto de las universidades, así como la implementación de sistemas de evaluación del desempeño, han traído consecuencias como un desfinanciamiento de las instituciones y una precarización laboral del profesorado, lo que va en detrimento de la educación. Se destaca que la aplicación de estas políticas ha encontrado resistencias a su paso a través de movimientos sociales, pero también con la creación de instituciones que tienen principios pedagógicos alejados del neoliberalismo. La selección de la literatura presentada se llevó a cabo a partir de una búsqueda en bases de datos a partir de los términos Neoliberalismo y América Latina, Neoliberalismo y Universidad y América Latina, Neoliberalismo y Educación Superior y América Latina, tanto en español como en portugués e inglés. Durante la revisión de los trabajos, que incluye artículos, capítulos de libros, libros, tesis y páginas de internet, se identificaron y sistematizaron las discusiones centrales y los resultados de cada uno. Posteriormente, se estableció cuáles han sido las principales temáticas señaladas por las y los autores. Como conclusión, se enfatiza la necesidad de que nuevos estudios de corte cualitativo profundicen en cómo el neoliberalismo se intersecta de forma particular con ciertas categorías sociales para configurar la experiencia de las personas al interior de las universidades.

\section{Palabras clave}

Neoliberalismo - Educación superior pública - Desfinanciar - Evaluar - Latinoamérica.

1- Agradecimientos: Quiero agradecer los comentarios que hicieron a este artículo Roselia Huerta y Serhat Tutkal. También agradezco profundamente las recomendaciones hechas por quienes evaluaron mi trabajo ya que contribuyeron a presentarlo de una mejor manera.

2- Universidad Nacional de Colombia, Bogotá, Colombia. Contacto: icastelaoh@unal.edu.co 


\section{Researches on the effects of neoliberalization of public higher education in Latin America}

\section{Abstract}

This article reviews the literature published between 1995 and 2019 addressing the effects of neoliberal policies being implemented in Latin American public higher education. It accounts for the ways university budget cuts as well as adopting performance evaluation systems resulted in defunding institutions and made professor posts precarious, which harmfully affected education. It is underlined that the imposition of these policies has faced resistance from social movements, and were also resisted by creating institutions with pedagogical principles other than the neoliberal ones. Selecting the literature presented was carried out by searching databases for the terms Neoliberalism and Latin America, Neoliberalism and University and Latin America, Neoliberalism and Higher Education and Latin America, in Spanish, Portuguese, and English. While revising the works, which includes articles, book chapters, books, dissertations, and web pages, the major discussion and results of each work were identified and systematized. Subsequently, the main themes highlighted by the authors were established. The conclusion found was the necessity of new qualitative studies, which would lead to an in-depth understanding how neoliberalism was intersected in particular ways with certain social categories in order to shape the experiences of people inside the universities.

\section{Keywords}

Neoliberalism - Public higher education - Defunding - Evaluation - Latin America.

\section{Introducción}

Este artículo se centra en presentar de manera sintética cuáles han sido las consecuencias que ha traído la aplicación de políticas neoliberales en la educación superior pública (ESP) en América Latina a partir de los estudios publicados entre 1995 y 2019. Para Harvey (2007), el neoliberalismo es una teoría de prácticas tanto políticas como económicas, para la cual la mejor forma de lograr el bienestar humano es permitir el libre desarrollo de las capacidades y de las libertades empresariales del individuo dentro de un marco institucional, creado y preservado por el Estado, caracterizado por derechos de propiedad privada fuertes, mercados libres y libertad de comercio. En ese sentido, es importante mencionar que el capitalismo es un sistema económico en donde los medios de producción se mantienen predominantemente en manos privadas que se benefician del trabajo asalariado gracias a la plusvalía, y en donde la vida económica se organiza de acuerdo con los principios del mercado. Por su parte, el neoliberalismo busca beneficiar al sistema capitalista ya que postula que la mejor forma en la que puede operar la economía 
es cuando el Estado no interviene en ella debido a que así, supuestamente, se posibilita la eficiencia, el crecimiento y la prosperidad generalizada. Por ello, entre sus políticas incluye la privatización, el bajo gasto público, la desregulación y la reducción de la provisión de asistencia social (HEYWOOD, 2013, 2017). Esto revierte en una mayor acumulación de capital para quienes poseen los medios de producción ya que, además de beneficiarse de recortes a impuestos, se hacen cargo de las actividades que antes eran una responsabilidad del Estado, como los servicios de salud, los servicios públicos y la educación, entre otros.

Más allá de ello, Brown (2005) enfatiza que el neoliberalismo es una racionalidad rectora que esparce los valores y las mediciones del mercado a cada esfera de la vida y que interpreta al ser humano exclusivamente como homo oeconomicus. Si bien el neoliberalismo configura la vida en términos económicos, políticos, sociales y culturales, tiene una paradoja como fenómeno global ubicuo y omnipresente: está desunificado y no es estable, por lo que varía geográfica y temporalmente (BROWN, 2005), con lo que es preciso mirar cuáles han sido sus efectos para la realidad latinoamericana.

Con ese propósito, en primer lugar se exponen cuáles han sido algunas de las consecuencias económicas y sociales que ha traído la aplicación de políticas neoliberales en América Latina. Con el objetivo de describir el estado de desarrollo alcanzado en lo referente a las consecuencias de la neoliberalización de la ESP, se presentan los resultados de algunos de los estudios que de 1995 a 2019 se han enfocado en analizar el hecho. Esta selección temporal obedece a que varios autores coinciden en señalar que es a principios de la década de los ochenta cuando se empiezan a poner en práctica políticas educativas de corte neoliberal en la región, con lo que las consecuencias de dichas iniciativas empiezan a ser estudiadas tres lustros después, llegando hasta nuestros días. Esto es importante porque la aplicación de las políticas neoliberales no ha sido estática ni ha tenido consecuencias inmediatas, sino que sus efectos han sido tangibles, y lo seguirán siendo, con el paso del tiempo, ya que sus principios siguen rigiendo en muchos de los países de América Latina.

La elaboración de esta revisión de literatura consistió en dos procesos: el primero incluye la búsqueda, la selección y la organización de las fuentes de información; y el segundo se trata de un análisis hermenéutico del contenido de las fuentes. La búsqueda se llevó a cabo a partir de los términos Neoliberalismo y América Latina, Neoliberalismo y Universidad y América Latina, Neoliberalismo y Educación Superior y América Latina en español, en portugués y en inglés. Las bases de datos consultadas fueron Google Scholar, Scielo, así como los repositorios de dos instituciones de educación superior latinoamericanas, El Colegio de México y la Universidad Nacional de Colombia. La búsqueda se centró en el término neoliberalismo, ya que, de acuerdo con Brown (2005), es el vocablo empleado por quienes critican dicha racionalidad, y el objetivo de este artículo de revisión es presentar los trabajos que den cuenta de los efectos negativos de su implementación en la región. Durante el análisis de la literatura, que incluye artículos, capítulos de libros, libros, tesis y páginas de internet, se identificaron aquellos trabajos que de manera puntual dieran cuenta de las repercusiones de la implementación de las políticas neoliberales en la ESP en América Latina, y posteriormente se sistematizaron las principales discusiones y resultados de cada trabajo. Finalmente, se estableció cuáles han sido las temáticas más recurrentes destacadas por las y los autores, que se presentan 
a continuación. Cabe señalar que este trabajo no pretende ser exhaustivo: su propósito, como se ha mencionado, es presentar un panorama general de las consecuencias que han sido enfatizadas por la literatura anterior.

La temática de la que me ocupo aquí ha sido estudiada desde diversos enfoques, que incluyen los ensayos monográficos y críticos, así como la revisión de literatura y el análisis de las legislaciones nacionales. En su mayoría, se han llevado a cabo estudios de corte cuantitativo que se centran en el análisis de datos estadísticos y de documentos presentados tanto por instancias oficiales como por organismos internacionales, resaltándose también la aplicación de encuestas. En una menor proporción, se han realizado estudios cualitativos que han empleado la observación, las entrevistas y los grupos focales para obtener su información empírica. Por ello, para profundizar en las consecuencias que estas políticas han tenido sobre las personas, se sugiere que en futuras investigaciones se empleen métodos cualitativos que permitan profundizar en las experiencias vividas.

A partir de la revisión de literatura, se subrayan como principales características de la neoliberalización de la ESP la reducción al presupuesto de las instituciones, así como el establecimiento de sistemas de evaluación del desempeño académico. En seguida, se señalan cuáles son las resistencias que el neoliberalismo ha encontrado, así como algunas propuestas de cambio. Finalmente, se concluye acentuando la necesidad de desarrollar investigaciones en las que se analice cómo el neoliberalismo se intersecta con ciertas categorías sociales dentro de la academia en América Latina para configurar la experiencia de las personas en su interior.

\section{Las consecuencias del neoliberalismo en América Latina}

Los efectos de la implementación de las reformas neoliberales en América Latina tienen diversas interpretaciones. En primer lugar, Leal Villegas (2016) enfatiza que no es posible hacer generalizaciones respecto al impacto que ha tenido la aplicación de las políticas neoliberales, dado que se debe considerar la estructura productiva y la manera en la que cada país se inserta en la economía mundial, así como sus reacciones ante el precio internacional del petróleo, la política monetaria estadounidense, y su inestabilidad política y financiera interna. Por su parte, Alburquerque Llorens (2009) apunta que la neoliberalización es una práctica alternativa de desarrollo en donde el sector público se flexibiliza, descentraliza y se orienta a buscar una interacción eficiente con sectores privados de la economía, en tanto Álvarez y otros autores (2009) señalan que se trata de una estrategia para abaratar la fuerza de trabajo. Así, un primer punto a subrayar es que hay percepciones diferenciadas en torno a los perjuicios y beneficios del neoliberalismo en la región, lo que puede deberse a que el neoliberalismo es dinámico y tiene diversas consecuencias dependiendo el contexto.

Fernández Jilberto y Riethof (2001) y Fiol (2002) señalan que la apertura al mercado mundial de bienes y capital, así como la desregulación de las economías nacionales, constituyen la segunda gran transformación de América Latina desde la crisis de los años treinta. Destacan que a final de la década de los noventa del siglo pasado casi todos los países de la región enfrentaron una clara reducción del crecimiento económico, y algunos 
hasta recesión, cuyo costo social se ve reflejado en los índices de pobreza e indigencia (CALVENTO, 2006), por lo que se le denominó la década perdida. Además, en casi todos los países disminuyeron las inversiones en los servicios públicos, como la educación y la salud. Por ejemplo, Torres Gonzáles (2009) emplea un enfoque histórico para señalar que en Colombia se ha privatizado el derecho a la educación de manera progresiva mediante cobros directos a las familias y con la contratación de servicios educativos privados a través de subsidios a la demanda. Esto ha provocado, siguiendo a Narodowski (2010), que la educación se encuentre diversificada, ya que, por una parte, está la educación monopolizada por el Estado, con problemas financieros, empobrecida en términos de capacidad educadora y dirigida a la población más pobre; y por otra, un sector privado que, en términos de eficiencia del gasto público, se hace funcional al sector estatal, con mayores márgenes de maniobra para educar y capacidad de estructurar proyectos autónomos, y dirigida a los sectores socioeconómicos de mayores recursos. En ese sentido, Pires y Reis (1999) señalan que en Brasil la estrategia del gobierno ha sido abandonar las instituciones de educación superior a su propio destino, hasta que, en el agotamiento y el estrangulamiento, acepten soluciones que van en detrimento de su función de producción y distribución democrática del conocimiento. En ese sentido, Lampert destaca que

Los últimos gobiernos, a partir de la década de los 90, además de no realizar las debidas inversiones ni implementar políticas públicas ajustadas a la realidad de la educación superior pública, están reduciendo los presupuestos, lo que afecta a la universidad en su conjunto, en lo concerniente a las funciones básicas de educación, investigación y difusión y pone en riesgo la operatividad, la manutención y la infraestructura de las instituciones federales de educación superior. (2005, p. 110).

En el caso de México, Ornelas Delgado (2009) señala que hay un detrimento en las universidades públicas debido a que, si bien la Ley de Ciencia y Tecnología señala que se debe invertir el 1\% del Producto Interno Bruto (PIB) en dichos ámbitos, el Estado únicamente otorga el 0,38\%. En el siguiente apartado se profundiza en estas cuestiones.

Ahora bien, Raza (2000) considera que la vulnerabilidad y el alto grado de dependencia de las economías latinoamericanas hacia los centros capitalistas es lo que impide un desplazamiento significativo de las relaciones de poder a favor de la periferia. En ese sentido, López Meraz y Villegas Loeza (2015) y Lorusso (2015) apuntan que seguir las medidas dictadas por el Fondo Monetario Internacional (FMI), el Banco Mundial (BM) y el Consenso de Washington -adoptadas tanto por gobiernos autoritarios como por los considerados democráticos con la consigna del fin del Estado (VILAS, 2010)- favorece una mayor dependencia hacia las decisiones del gobierno estadounidense, deviniendo en desigualdad e injusticia social, con una población que no logra incorporarse a los ciclos de producción de capital, incrementando el desempleo y la informalidad en donde el crimen organizado y la delincuencia logran captar recursos.

Ibarra (2011) encuentra que prevalecen un menor crecimiento, la desindustrialización, la concentración de ingresos y los empleos precarios. Gaviria Ríos (2004), Julián Vejar (2017) y Soares Campos (2017) señalan el aumento de la subcontratación y del desempleo, 
siendo el origen de la propagación del trabajo informal y de la destrucción de sindicatos, así como del déficit fiscal (GARCÉS CORREA et al., 2017; MORA CORTÉS, 2015; VILLAMIZAR MARULANDA; URIBE ARBELÁEZ, 2009). Con ello, señalan Portes y Hoffman (2003), los empleadores grandes y medianos, los altos ejecutivos y los profesionales conforman la clase dominante en todos los países latinoamericanos (con excepción de Cuba). En ese sentido es importante señalar que América Latina es una de las zonas de mayor desigualdad social, en donde el 10\% más rico de la población recibe entre un 40\% y un $47 \%$ del ingreso nacional, en tanto el 20\% más pobre solo percibe entre el $2 \%$ y el $4 \%$ (FERREIRA; WALTON, 2005), desatando la disparidad entre quienes se ven beneficiados y a quienes ha perjudicado la instauración de las reformas neoliberales. Esta situación ha sido naturalizada bajo el argumento del esfuerzo individual (MAÑUNGA ARROYO, 2015), es decir, se busca normalizar la condición de desigualdad al afirmar que quienes perciben más ingresos han trabajado de manera ardua para lograr lo que tienen, en tanto que aquellas personas con los menores ingresos no se han esforzado ni trabajado lo suficiente para modificar su situación.

Además de lo anterior, a partir de la aplicación de políticas económicas neoliberales, también se ha presentado una ingobernabilidad por el surgimiento de movimientos sociales de protesta que demandan sus derechos (DE SOUSA SANTOS, 2018; KATZ, 2012; LÓPEZ CASTELLANOS, 2016; NAVARRETE SAAVEDRA, 2010; RAMÍREZ, 2016; SANTANA CASTILLO, 2014). Asimismo, no hay un control ni prácticas que obliguen a los gobiernos a rendir cuentas (CONAGHAN; WOLFSON; MALLOY, 1997), con lo que hay corrupción e injusticia (LARA; LÓPEZ, 2002; LEVINE, 1992).

A grandes rasgos, los trabajos anteriores permiten tener una visión general de lo que ha implicado la aplicación de políticas neoliberales en un contexto como el de América Latina, es decir, una región que pertenece al sur global, marcada por la corrupción, la violencia estatal y los gobiernos autoritarios, en donde su principal actividad económica ha sido la extracción de materias primas en beneficio de los grandes países industrializados, por mencionar solo algunas características. Así, en este contexto específico, la cada vez mayor ausencia del Estado ha generado precarización, informalidad, desempleo, pobreza, desigualdad, corrupción, criminalidad e injusticia. A continuación, se presentan las consecuencias particulares que el proyecto neoliberal ha traído para la educación, específicamente en el ámbito universitario.

\section{Efectos de la neoliberalización de las universidades públicas}

El neoliberalismo es un conjunto dinámico de políticas que han buscado ser aplicadas con el objetivo de lograr el aumento de la productividad y de la competitividad de acuerdo a la economía de mercado (RODRIGUEZ PECIAR, 2009). Escalante Gonzalbo (2015) apunta que para los neoliberales es necesario abrir el mercado de la educación, diversificar las oportunidades de aprendizaje e incentivar a que quienes estén calificados compartan sus conocimientos. Castro (1997) elabora un ensayo en el que destaca que la formulación 
de las políticas educativas de corte neoliberal puestas en marcha en América Latina desde principio de los 80 se han sustentado en un reduccionismo técnico-economicista, trayendo como consecuencias la reconversión y flexibilización del financiamiento, la descentralización del sistema de educación y el impulso a la expansión de las escuelas privadas. Es importante apuntar que la implementación de políticas neoliberales ocurre de manera paulatina, por lo que las consecuencias presentadas han acaecido a partir de procesos dinámicos y complejos en los que confluyen diversos factores. Por ejemplo, Borges y Fernández Aquino (2012) subrayan que en Brasil el desmantelamiento del aparato científico-tecnológico, así como el empresariamiento de la educación superior inició desde la década de 1990 con Fernando Collor de Mello. Posteriormente, los gobiernos de Fernando Henrique Cardoso (1995-2002), caracterizados por una fuerte mercantilización de la sociedad, buscaron reestructurar las instituciones públicas con miras a la privatización manteniendo un modelo educativo deseado por los sectores conservadores de la sociedad (BENATTI; MUSTAFA, 2017; DE SOUZA LIMA, 2011; LIMA; AZEVED0; CATANI, 2008). Finalmente hay una mayor sistematización de este proceso durante el gobierno de Luiz Inácio Lula da Silva, aunque a la par también se elaboraron propuestas con miras a generar un cambio institucional (TEODORO; GUILHERMINA, 2017). En ese sentido, Sguissardi y Silva Jr. (2012) mencionan el Programa de Apoyo al Plan de Reestructuración y Expansión de las Universidades Federales (REUNI), la Educación a Distancia (EAD), la Universidad Abierta de Brasil (UAB), la continuidad del desarrollo del posgrado y el fomento a la investigación que allí se lleva a cabo como programas que si bien han permitido la expansión de la ESP en Brasil, también han devenido en una mercantilización del conocimiento y en una certificación, sobre todo de los programas de posgrado, en masa.

Entonces, hay una variedad de políticas que se han implementado y modificado en el tiempo, pero a partir de la literatura previa es posible señalar que se destacan dos en América Latina: a) el recorte al financiamiento de las instituciones públicas y b) la implementación de sistemas de evaluación del desempeño académico. Ambas medidas han resultado perjudiciales para el conocimiento y para la vida de quienes integran las universidades debido a que hay presiones internas tanto para conseguir recursos externos que permitan llevar a cabo investigaciones, como para alcanzar los estándares planteados por los sistemas de evaluación. Respecto a esto último, Zapata y Tejeda, a partir de una revisión de literatura, enfatizan que todavía no hay

[...] un método suficientemente robusto como para identificar si los cambios generados por el aseguramiento de la calidad se han traducido efectivamente en mejoras en la enseñanzaaprendizaje. Por otro lado, tampoco se puede caer en una postura naïve y asumir que las acciones implementadas en respuesta a las recomendaciones externas conducen inmediatamente a la superación de los problemas académicos de las universidades. (2009, p. 204).

De esta manera, las discusiones acerca de los beneficios y los perjuicios de la implementación de políticas neoliberales en la ESP aún están abiertas. 


\section{El recorte al presupuesto universitario}

Siguiendo a De Sousa Santos (2007), la crisis institucional que han enfrentado las universidades desde hace treinta años ha sido provocada por su desfinanciamiento y su descapitalización, a causa de su pérdida de aseguramiento como bien público por parte del Estado. Borón (1995) expone, basado en cifras oficiales, que las universidades públicas en América Latina presentan una crisis de financiamiento asentada en las restricciones económicas de los sistemas educativos. Entre las pautas implementadas en la región para crear un mercado educativo encuentra el recorte al presupuesto universitario, evidente en los reducidos montos que el Estado otorga a la educación, y el ímpetu de las políticas privatizadoras del sistema educacional. Ello ha traído condiciones negativas tanto para el profesorado como para el estudiantado, debido a que, como Thompson (1995) apunta a partir del análisis de declaraciones, informes y documentos producidos por distintas organizaciones internacionales acerca de la educación superior pública en Costa Rica, hay una fuerte contradicción entre las demandas de proporcionar acceso a la educación en forma equitativa y de alta calidad, y el alto compromiso financiero requerido para ello. En ese sentido, Diodati y Mendíaz (2010), a partir de la revisión de las publicaciones del BM en torno a las políticas recomendadas para Ciencia y Tecnología, enfatizan que no es el exceso de autonomía lo que impide a las universidades públicas cumplir su compromiso social, sino la falta de medios financieros para desarrollar las nuevas funciones que exige la sociedad del conocimiento, punto suscrito por Stromquist (2012). En ese sentido, Aboites (2012) señala que a principios de los noventa, universidades públicas en México como la UNAM enfrentaron una reducción en su financiamiento de hasta un 37\%, situación que ha variado en el tiempo.

López Guerra y Flores Chávez (2006) destacan que, bajo el argumento de la mejoría de la calidad educativa mediante la competencia, lo que ha ocurrido es que ha habido un traslado del financiamiento público al ámbito privado, privatizando así la educación. Ejemplo de ello es el programa colombiano Ser pilo paga en donde el Estado ha transferido, a partir de su política de subsidios a la demanda, recursos a instituciones privadas en detrimento de los recursos otorgados a las universidades públicas (RAMOS RUIZ; PARRA RAMÍREZ, 2017). Con las restricciones económicas, muchas de las universidades públicas, que viven una combinación de privatización y de control gubernamental (DAUTREY, 2017; TORRES; SCHUGURENSKY, 2001), deben autofinanciarse, de preferencia por la vía de las matrículas y los aranceles, lo que parece ser una tendencia mundial (ZAPATASEPÚLVEDA; JIMÉNEZ-BENÍTEZ; CONCHA-ASTORGA, 2013). En ese sentido, también existen universidades con posiciones ambiguas, como las comunitarias de Brasil, "[...] definidas como públicas de derecho privado, es decir, gozan de filantropía, su gestión es colegiada, los alumnos pagan mensualidades, sin embargo, el patrimonio adquirido es público" (NETTO MACHADO, 2008, p. 39). De esta manera, las universidades públicas enfrentan lo que Acosta Silva (2002a) define como neointervencionismo por el gran número de regulaciones impuestas por los gobiernos nacionales a los servicios de educación superior. Vega Cantor (2011), a partir del análisis de fuentes directas oficiales, comunicados oficiales, entrevistas, informes de prensa oficial y rastreo documental, señala 
que la autofinanciación de las instituciones tiene el objetivo de convertir a la educación universitaria en un negocio, situación que ha traído como consecuencia que haya una pérdida en el nivel académico, superpoblación, edificios dañados, así como bibliotecas desactualizadas y laboratorios inadecuados. "Además, ha aumentado la flexibilización docente, con la reducción de los profesores de planta y la generalización de los catedráticos, mal pagos y sin ningún incentivo para permanecer en las universidades" (VEGA CANTOR, 2011, p. 347). Así, el desfinanciamiento afecta de manera directa al profesorado, pero también a las personas que desean continuar con su formación.

\section{La precarización laboral y las restricciones en el acceso a la educación}

Con la escasez de recursos, las condiciones del profesorado se caracterizan por una carga de trabajo más intensa debido a que deben ocuparse de un mayor número de actividades y atender grupos cada vez más numerosos (DIAS SOBRINHO, 2005; MAIA, 2019); contratos a corto plazo que permiten ahorrar recursos al pagar solamente por las horas de clase dictadas (SANTOS, 2008); inseguridad laboral; competitividad excesiva; desequilibrio de poder entre las directivas y el personal académico, así como un debilitado poder sindical (MARTÍNEZ ALCÁNTARA; PRECIADO SERRANO, 2010). Todo ello conforma lo que puede ser considerado como precarización laboral, aunque como se expone más adelante, la precarización también es moral.

Además de ello, esta desfinanciación también tiene graves consecuencias directas para el estudiantado, ya que, como destacan Hernández Gutiérrez (2016) y Santarrone y Vittor (2004), la educación se convierte en un producto del mercado al que tienen mayor posibilidad de acceso quienes cuentan con capital económico y cultural, con lo que las instituciones devienen más excluyentes (ALCÁNTARA, 2008; GENTILI, 1998). Aboites (2008) apunta que la universidad pública, acompañada de la restricción a la matrícula y el uso de exámenes estandarizados vinculados a los sistemas de evaluación y acreditación, se ha convertido en un filtro para excluir del acceso a empleos y mejor calidad de vida a los grandes sectores sociales populares, afectando así la igualdad de oportunidades, especialmente en Nicaragua, Argentina, Chile y Brasil (ARNOVE et al., 1998). Con ello, siguiendo a De Sousa Santos (2007), la universidad se ha transformado de un servicio al que se tenía acceso por vía de la ciudadanía, al que ahora se tiene acceso por la vía del consumo, mediante el pago. Con la eliminación de la gratuidad y la sustitución de becas de estudio por préstamos, que se han convertido en la única posibilidad que muchas personas encuentran para poder ingresar y permanecer en la universidad (GALIND0; GÓMEZ; RODRÍGUEZ, 2015), el estudiantado se transforma de ciudadanos en consumidores, todo ello bajo el paradigma de la educación centrada en el individuo y en la autonomía individual. Así, el ethos público de la universidad se ha desplazado hacia otro más corporativo y privatizante, en el que la educación es considerada un bien de consumo y no un derecho (FARAH, 2007), con lo que también ha habido una proliferación de instituciones privadas. Por ejemplo, en Brasil el sistema privado creció un 151.6\% de 1996 a 2004 (BENATTI; MUSTAFA, 2017; BORGES; FERNANDEZ AQUINO, 2012; COSTA; GOULART, 2018; DIAS SOBRINHO, 2010; DOURAD0, 2002), con lo que 
casi el 90\% de las instituciones de educación superior en el país pertenecen al sector privado (DIAS SOBRINHO, 2013), debido a que conglomerados de empresas y sociedades de cartera se interesaron en invertir en este sector (SERAFIM, 2011). Pero la existencia de una mayor cantidad de universidades privadas no necesariamente implica que haya una mayor cobertura: Dias Sobrinho (2013) subraya que únicamente el 15\% de las y los jóvenes entre 18 y 24 años forma parte de la matrícula de la educación superior en Brasil.

\section{La implementación de sistemas de evaluación del desempeño}

Acosta Silva (2002b) señala que desde la década de los noventa aparecieron en América Latina políticas públicas basadas en sistemas de evaluación y acreditación de la calidad y la excelencia con la finalidad de formar élites científicas, intelectuales y profesionales en la sociedad mundial. Así, el viejo paradigma desarrollista de intervención discreta del Estado que patrocinaba y respetaba la autonomía universitaria, se modernizó a partir de la evaluación del desempeño y la búsqueda de calidad, con lo que el Estado tomó centralidad en las reformas que buscan cambios en el financiamiento público. Un punto fundamental de la evaluación es que se establece como la condición primordial para el financiamiento y la legitimidad social de la universidad (SILVA MONTES, 2011).

El objetivo de establecer sistemas cuantitativos estandarizados de evaluación de la calidad del conocimiento, similares a los que se emplean en sectores de finanzas, producción y comercio, es hacer más eficiente la producción del conocimiento (CAMPILLO, 2015). Este sometimiento a indicadores de calidad del conocimiento, que se basan en instrumentos presumiblemente objetivos y cuantificables, supuestamente permite que se apliquen a toda persona, actividad e institución para determinar su excelencia, presuponiendo que en todo el mundo las condiciones materiales de las universidades y de las personas son iguales. Esta pretendida estandarización de la calidad deviene en jerarquizaciones porque las formas de producción y transmisión del conocimiento son variables de acuerdo a las disciplinas y a los recursos financieros que se tengan para ello. Así, se han generado distinciones en la propia universidad (entre las ciencias y las ingenierías, por un lado, y las artes y las humanidades, por el otro); al interior de cada país (entre las nuevas élites globalizadas y el nuevo precariado universitario con sobretitulación); y entre países (entre las potencias intelectualmente hegemónicas y las periféricas o subalternas) (CAMPILLO, 2015).

Jiménez Ortiz (2011) afirma que los discursos de evaluación y acreditación de la calidad de organismos internacionales como el BM, la OCDE, la CEPAL, además de la UNESCO, tienen un papel hegemónico en la definición de la agenda interna de las políticas educativas de los países latinoamericanos (DE SOUZA LIMA, 2011; LEITE; GENRO, 2012), buscando la legitimación de las estructuras, los mecanismos, las nociones y las visiones del poder económico y político mundial, sin tener presente el contexto regional. Se espera que países en vías de desarrollo cumplan con las mismas metas que logran los países desarrollados, lo cual resulta imposible por el hecho de que los recursos financieros invertidos en educación no son equiparables. Por tanto, el modelo impuesto no se adapta a las condiciones sociohistóricas y pretende debilitar el financiamiento público para que 
el sector privado se haga cargo (ARIAS CAMPOS; ROMERO BARBOZA, 2018; GALINDO; GÓMEZ; RODRÍGUEZ, 2015). Así, Gallardo Córdova y otras autoras (2015), a partir de un estudio cuantitativo realizado con docentes de México, Colombia, Costa Rica, Ecuador, Guatemala y Perú, destacan que las prácticas de evaluación se orientan al cumplimiento de dichos estándares internacionales que no corresponden con las realidades nacionales. Dias Sobrinho (2010) señala que en Brasil los exámenes nacionales son usados para medir la eficiencia y la efectividad de la educación de acuerdo con los criterios y necesidades de los estados neoliberales; en ese sentido, destaca que el Examen Nacional de Cursos, que se hizo popular como Provão, obligatorio para que las y los estudiantes pudieran graduarse, se estableció en 1995 y gradualmente se convirtió en el instrumento central para la evaluación de la educación superior brasileña desde 1996 hasta 2003. Posteriormente, se implementó el Sistema Nacional de Evaluación de la Educación Superior (SINAES), que incluye el Examen Nacional del Desempeño del Estudiante (ENADE), usado como una herramienta de evaluación para diagnosticar las habilidades académicas y profesionales que el estudiantado puede demostrar en distintos momentos de su carrera, siendo un componente del sistema de evaluación de la educación superior en dicho país.

\section{La mercantilización del desempeño docente}

A partir de la evaluación del desempeño, siguiendo a Escalante Gonzalbo (2015), se han instaurado tres acciones de lógica empresarial que, como se aprecia, están basadas en la premisa del mérito individual estimulado por el beneficio económico, formando sistemas de recompensas económicas por productividad académica (BONIFAZ CHIRINOS, 2018):

1) Establecer estándares de productividad, que suelen ser índices numéricos, cuya finalidad es hacer comparaciones que permitan medir la eficiencia del profesorado y al mismo tiempo provocar la competencia.

2) Simular mercados a partir de sistemas de salarios flexibles (ARNOVE et al., 1998; FASSBENDER; SILVA DÍAZ, 2014) asociados a alguna clase de rendimiento, sistemas de estímulos, bonos de productividad y/o fondos especiales sujetos a competencia, con la finalidad de estimular la producción de conocimiento.

3) El avance profesional se supedita a la evaluación cuantificable del desempeño, lo que también se refleja en el sueldo, medida que también busca incrementar la productividad.

$\mathrm{Al}$ ser uno de sus principales objetivos la evaluación estandarizada de la eficiencia, el neoliberalismo ha provocado que las demandas de publicación para el profesorado se incrementen (publish or perish). Ante ello, se presenta un contexto altamente competitivo, en donde, a pesar de que las instituciones no cuentan con los recursos suficientes para satisfacer las necesidades de recursos para la producción de conocimiento, se les exigen la medición de dicha producción que, como destaca Gómez-Morales,

[...] se constituye en forma privilegiada porque le permite a aquella politica poner en marcha sus procesos de rendición pública de cuentas (accountability) y sus rituales de verificación, en torno de los cuales gira su credo de excelencia, transparencia, decisión informatizada, emprendimiento, competitividad. (2017, p. 16). 
$\mathrm{Al}$ estar en permanente evaluación del desempeño, surge como una característica de la neoliberalización de la universidad la sensación de inestabilidad, que desencadena una interacción humana conflictiva y difícil, con episodios de burnout, agotamiento, despersonalización y falta de realización personal (MARTÍNEZ ALCÁNTARA; PRECIADO SERRANO, 2010). La docencia universitaria es vista como una función, objeto de revisión y evaluación, que debe responder a requerimientos de calidad (LONDOÑO OROZCO, 2015), con lo que todas las complejidades, los desafíos, las condiciones, las posibilidades, los saberes y las herramientas de la enseñanza no se tienen en cuenta, con lo que hay una desvalorización de la docencia (GENTILI, 2001).

Por ello resulta imprescindible entender la precariedad no únicamente en relación con las condiciones laborales, sino como una práctica encaminada a la construcción de una subjetividad marcada por la inseguridad vital, con un sometimiento constante a procesos de evaluación en donde la calidad de los trabajos realizados no tiene relevancia porque prima la cuantificación de los mecanismos de evaluación (ÁVILA; AYALA; GARCÍA, 2018). Así, la precarización moral se establece como gubernamentalidad neoliberal a partir de la evaluación del desempeño, la hipervigilancia, la inversión empresarial del individuo, y el centrar la subjetividad en el rendimiento, la acumulación y la competencia (PÉREZ; MONTOYA, 2018).

Allí el mercado opera definiendo, a través de burocracias científicas muy particulares, que el criterio de legitimidad para permanecer y tener un lugar reconocido en la academia, no es solo publicar en revistas reconocidas, sino publicar mucho, lo que diversidad de autores van a llamar un estándar de productividad regulado por una cultura de la auditoría desde la que se configura una relación de poder entre quien define qué es legítimo y aquel que busca legitimidad. (PÉREZBUSTOS, 2019, p. 37).

Con ello, el desempeño docente es medido a partir de la cantidad de productos académicos que genera, sin tener presente la calidad de los trabajos ni su labor con el estudiantado.

\section{La pedagogía al servicio del mercado}

Una consecuencia más de la evaluación del desempeño es el afianzamiento de una corriente dominante de la pedagogía constructivista universitaria, cuyo interés son los resultados efectivos de aprendizaje que formen profesionales emprendedores y capaces de resolver problemas (SILVA MONTES, 2011); bajo esta nueva lógica, en donde lo que premia es el desempeño y la capacidad operativa, al profesorado se le convierte en un mero ayudante del estudiantado (MARTÍNEZ B00M, 2000). Con ello, hay la imposición de un currículo basado en competencias, caracterizado por el saber hacer laboral con una homogenización del conocimiento, señalan De la Cruz (2012) y Espinoza (2017). Esto tiene como resultado la pérdida del concepto de "bien público" de la universidad al satisfacer demandas privadas e individuales, dejando de lado las potencialidades laborales 
del estudiantado y los requerimientos del desarrollo de los países. Con ello, apunta Ribeiro (2000) en su ensayo basado en Chile, Argentina y Brasil, el pensamiento crítico y la libertad necesaria para la producción de conocimiento se han visto socavadas debido a que se ha sometido a las universidades públicas a la lógica empresarial, con lo que ciertas disciplinas pueden estarse convirtiendo en abastecedoras de capital humano especializado al acoplarse a las necesidades de las grandes corporaciones multinacionales (BONITO, 2016). Con ello, el acceso a la universidad puede estar determinado por la obtención de competencias y calificaciones que se asocian con los beneficios en los mercados de trabajo y en los perfiles trazados por la estratificación social, apunta Vizcaíno (2005); es decir, pueden ingresar a la universidad en mayor cantidad quienes serían más útiles al mercado.

Así, tomando como referencia cifras, anuarios y discusión teórica, Arnove y otros autores (1998) destacan que los métodos de enseñanza y aprendizaje se basan en suposiciones de leyes universales, deviniendo en currículos desfasados, en donde las emociones se ven principalmente como obstáculos, generando relaciones profesorado-alumnado donde el cuidado y el respeto son considerados inútiles por no ser cuantificables ni redituables. De esta forma, subrayan Pérez-Bustos y Botero-Marulanda (2014), la ciencia eficiente, inmersa dentro del sistema capitalista en donde el neoliberalismo es su principal corriente, no considera como prioritarios el ser con los otros y el construir vínculos vitales.

Es así como tanto el recorte al presupuesto de las universidades como la implementación de sistemas de evaluación del desempeño académico han minado no solamente a las instituciones, sino que han devenido en condiciones laborales y académicas adversas con consecuencias negativas para la salud y el bienestar de las personas. No obstante, la literatura revisada da cuenta de cómo en Latinoamérica la oleada neoliberal ha encontrado resistencias a su paso, tanto en movilizaciones sociales como en nuevas propuestas, sobre todo pedagógicas, para hacer frente a los estragos desarrollistas.

\section{Resistencias y propuestas de cambio}

Cuando se habla de resistencia al neoliberalismo, se suele pensar y estudiar movimientos sociales masivos (ALCÁNTARA; LLOMOVATTE; ROMÃO, 2013; BERTELYBUSQUETS, 2016; GARCÍA; ORTIZ-CONTRERAS, 2016). Así, Díaz Escoto (2007), a partir de datos históricos y cifras oficiales, señala que la huelga estudiantil en la UNAM (19992000), en contra de la privatización y en defensa de la gratuidad de la educación superior, impidió el aumento de las cuotas y evitó el elitismo en la universidad. De Moraes Freire (2008) destaca que a partir de 2007 surgió un nuevo movimiento estudiantil en Brasil, principalmente en contra de la Reforma Universitaria y los escándalos de corrupción. En ese sentido, Bonifacio (2012) describe las tres tomas realizadas por el estudiantado en los años 1995, 2004 y 2006 en la Universidad Nacional del Comahue (Argentina) en oposición a las pretensiones privatistas que, según el movimiento estudiantil, propician la apertura de desigualdades políticas, sociales y culturales. Cruz Rodríguez (2013) apunta que en 2011 se llevó a cabo en Colombia una movilización estudiantil que logró que se retirara el proyecto de ley que se centraba en la atracción de la inversión privada en las universidades públicas y el establecimiento de Instituciones de Educación Superior con 
ánimos de lucro, con lo que consiguieron la reivindicación de la educación como derecho y no como mercancía, aunque a finales de 2018 se llevó a cabo un gran paro estudiantil para exigir una mayor presupuesto para la ESP en el país, lo que reafirma que no hay un estatismo ni en la implementación de políticas neoliberales ni en las reacciones ante ellas. Así, Durão (2015) afırma que las movilizaciones sociales son una importante forma de actuar políticamente en contra de los cambios que promueven la mercantilización de la universidad porque logran frenar o aminorar la implementación de los preceptos neoliberales. Pero las movilizaciones sociales no han sido la única forma de resistencia.

Aboites (2008) indica que desde fines de los noventa y primeros años del siglo veintiuno, las luchas en el campo educativo habían comenzado a generar visiones distintas y proyectos concretos de una nueva educación y universidad en contra del neoliberalismo, como lo son la pedagogía desarrollada por el movimiento Sem Terra en Brasil, el proyecto de la Universidad de las Madres de la Plaza de Mayo en Argentina, la Universidad de la Tierra en México, así como las universidades comunitarias en Bolivia y las universidades interculturales en Ecuador. Massé Narváez (2008) coincide en señalar que estos proyectos surgen por el deterioro que el neoliberalismo provocó en la autonomía, así como por el fortalecimiento de las estructuras de poder burocrático en las instituciones. Morales Herrera (2012) encuentra que hay prácticas de resistencia del profesorado que pueden ser públicas, a partir de su participación en asociaciones o discusiones en espacios formales como comités o consejos de facultad; ocultas, sobre todo en el espacio del aula a través de contenidos y reflexiones críticas; e inclusive, desobediencia por parte de quienes cuentan con un contrato estable.

Respecto a las propuestas de cambio, Mora Moraga y Gutiérrez Espeleta (2015) proponen una transformación que busque la equidad basada en el bien común, así como defender la universidad pública a partir de la acción social. Para Durão (2015) es fundamental actuar políticamente, tanto institucionalmente como a través de movilizaciones colectivas, considerando la diversidad de sujetos políticos con sus diferentes propuestas educativas (MELO, 2005), es decir, evitar las estandarizaciones y las imposiciones de regímenes educativos en contextos que son diversos. Para González Luna (2017), el profesorado debe ejercer resistencia a partir de la criticidad, la prospección, la colaboración y la simbolización. Para ello, Miñana Blasco y Rodríguez (2003) sugieren una educación más vinculada y comprometida con su entorno, más abierta a diferentes tipos de saberes y de voces, más preocupada por su pertinencia y por la calidad de vida que por la competitividad y el control.

En ese sentido, Méndez Reyes y Morán Beltrán (2012) sostienen que es necesario un proceso de descolonización del saber y del ser para lograr la nueva transformación universitaria. Para Edelstein (2012), es preciso que el mejoramiento en la calidad de los procesos de producción, transmisión y apropiación del conocimiento tenga en cuenta las problemáticas sociales actuales, no los intereses del mercado. Sumado a ello, Rivero Bottero (2013) apunta que la educación debe verse como un espacio para ofrecer contención, herramientas intelectuales, afectivas y sociales, en donde aún quepa la solidaridad. Finalmente, Maceira Ochoa $(2006,2008)$ propone la pedagogía feminista como una forma de transformar a la sociedad a partir de la eliminación cultural y política de la opresión, 
la exclusión social, la violencia, la discriminación y el neoliberalismo, a favor de la democracia, los derechos humanos, la paz y la ecología.

\section{Reflexiones finales}

Los estudios anteriores permiten señalar que la adopción de políticas neoliberales en la ESP en América Latina ha traído como consecuencias el desfinanciamiento de las instituciones y la implementación de regímenes de evaluación que fomentan la competencia según la economía del mercado (RODRIGUEZ PECIAR, 2009), causando precarización laboral y moral en quienes integran la universidad, así como exclusión de quienes no poseen capitales económico y cultural. De esta manera, las políticas neoliberales han resultado, y siguen siendo, perjudiciales para quienes hacen parte de la academia latinoamericana.

Uno de los principales problemas de las políticas neoliberales basadas en el mérito es que presuponen que las condiciones materiales, sociales y culturales no tienen ningún efecto en la competencia entre las personas ya que el mercado lo regula todo de manera eficiente al ser ciego a cualquier distinción, cuando en realidad las distinciones sociales configuran las experiencias de las personas. En ese sentido, se destaca que en la región aún no se ha profundizado lo suficiente para indagar cómo la neoliberalización de la universidad configura las vivencias y las prácticas de quienes la integran; además, cómo ello se entreteje con otros elementos de diferenciación y distinción social que agravan la situación para ciertos sujetos sociales (generizados, racializados, estratificados, etcétera), con lo que futuras investigaciones podrían interesarse por dar cuenta de cómo los resultados de la neoliberalización de la ESP afectan de formas particulares a las personas a partir de las distintas identidades sociales intersectadas. Ante ello, es imprescindible indagar en cómo el neoliberalismo dentro de la academia se intersecta con el género, la raza, la clase social, etcétera, para marcar las experiencias de las personas en su interior.

\section{Referencias}

ABOITES, Hugo. El futuro de la universidad en América Latina. Revista Acción Educativa, Madrid, v. 8, p. 6-16, 2008.

ABOITES, Hugo. El derecho a la educación en México: del liberalismo decimonónico al neoliberalismo del siglo XXI. Revista Mexicana de Investigación Educativa, Ciudad de México, v. 17, n. 53, p. 361-389, 2012.

ACOSTA SILVA, Adrián. El neointervencionismo estatal en la educación superior en América Latina. Sociológica, Ciudad de México, v. 17, n. 49, p. 43-72, 2002a.

ACOSTA SILVA, Adrián. Poder y políticas universitarias en América Latina: el neointervencionismo estatal. Nueva Sociedad, Buenos Aires, v. 179, p. 32-44, 2002b.

ALBURQUERQUE LLORENS, Francisco. La crítica situación de América Latina al inicio de los noventa y la necesidad de una estrategia de desarrollo alternativa al neoliberalismo. América Latina Hoy, Salamanca, v. 4, p. 57-68, 2009. 
ALCÁNTARA, Armando. Políticas educativas y neoliberalismo en México: 1982-2006. Revista Iberoamericana de Educación, Madrid, n. 48, p. 147-165, 2008.

ALCÁNTARA, Armando; LLOMOVATTE, Silvia; ROMÃO, José Eustaquio. Resisting neoliberal common sense in higher education: experiences from Latin America. International Studies in Sociology of Education, London, v. 23, n. 2, p. 127-151, 2013.

ÁLVAREZ, Nacho et al. Ajuste y salario: las consecuencias del neoliberalismo en América Latina y Estados Unidos. Madrid: FCE, 2009.

ARIAS CAMPOS, Luis Diego; ROMERO BARBOZA, José Pablo. La mercantilización de la educación en Costa Rica: las universidades públicas en la mira de los organismos internacionales. Humanidades, San José, v. 9, n. 1, p. 1-34, 2018.

ARNOVE, Robert et al. La sociología política de la educación y el desarrollo en Latinoamérica: el Estado condicionado, neoliberalismo y política educativa. Revista de Educación, Madrid, v. 316, p. 85-107, 1998.

ÁVILA, Débora; AYALA, Ariadna; GARCÍA, Sergio. La Universidad y la vida..., o cómo mantenernos vivos en medio de la neoliberalización de la Universidad. Revista de Dialectología y Tradiciones Populares, Madrid, v. 73, n. 1, p. 55-61, 2018.

BENATTI, Lucimara Perpétua dos Santos; MUSTAFA, Patrícia Soraya. Privatização e precarização da política de educação superior no Brasil: impactos para a formação profissional em serviço social. Temporalis, Londrina, v. 16, n. 32, p. 141-158, 2017.

BERTELY-BUSQUETS, María. Políticas neoliberales y afectaciones territoriales en México. Algunos "para qués" de "otras" educaciones. LiminaR, Tuxtla Gutiérrez, v. 14, n. 1, p. 30-46, 2016.

BONIFACIO, José Luis. Neoliberalismo y movimiento estudiantil en la Universidad Nacional del Comahue. Cuestiones de Sociología, Buenos Aires, v. 8, p. 1-10, 2012.

BONIFAZ CHIRINOS, Mónica Patricia. Recompensas económicas en la academia: análisis de literatura empírica sobre el pago por mérito en universidades de siete países. Educación, Lima, v. 27, n. 52, p. 7-25, 2018.

BONITO, Justo Cuño. La universidad Latinoamericana en la encrucijada: amenazas, desafíos y soluciones. Revista Historia de la Educación Latinoamericana, Tunja, v. 18, n. 28, p. 241-277, 2016.

BORGES, Maria Célia; FERNANDEZAQUINO, Orlando. Educação superior no Brasil e as políticas de expansão de vagas do Reuni: avanços e controvérsias. Educação, Rio Claro, v. 22, n. 39, p. 117-137, 2012.

BORÓN, Atilio A. La economía política de la educación superior en América Latina: reflexiones desde el caso argentino. Perfiles Educativos, Ciudad de México, v. 69, p. 21-33, 1995.

BROWN, Wendy. Undoing the demos: neoliberalism's stealth revolution. New York: Zone Books, 2005.

CALVENTO, Mariana. Fundamentos teóricos del neoliberalismo: su vinculación con las temáticas sociales y sus efectos en América Latina. Convergencia, Toluca, v. 23, n. 41 ,p. 41-59, 2006. 
CAMPILLO, Antonio. La universidad en la sociedad global. Isegoría, Madrid, n. 52, p. 15-42, 2015.

CASTRO, Inés. El pragmatismo neoliberal y las desigualdades educativas en América Latina. Revista Mexicana de Sociología, Ciudad de México, v. 59, n. 3, p. 189-205, 1997.

CONAGHAN, Catherine M.; WOLFSON, James M.; MALLOY, Leandro. Democracia y neoliberalismo en Perú, Ecuador y Bolivia. Desarrollo Económico, Buenos Aires, v. 36, n. 144, p. 867-890, 1997.

COSTA, Camila Furlan da; GOULART, Sueli. Capitalismo acadêmico e reformas neoliberais no ensino superior brasileiro. Cadernos EBAPE.BR, Rio de Janeiro, v. 16, n. 3, p. 396-409, 2018.

CRUZ RODRÍGUEZ, Edwin. La reforma de la educación superior y las protestas estudiantiles en Colombia. Revista POSTData, Buenos Aires, v. 18, n. 1, p. 51-71, 2013.

DAUTREY, Philippe. Neocorporativismo y calidad en la educación superior pública mexicana. Revista de Investigaciones Políticas y Sociológicas, Santiago de Compostela, v. 16, n. 2, p. 65-82, 2017.

DE LA CRUZ, Silvia. La mercantilización de la educación en el contexto universitario. Revista Electrónica de Psicología Política, San Luis, v. 9, n. 28, p. 48-54, 2012.

DE MORAES FREIRE, Silene. Movimento estudantil no Brasil: lutas passadas, desafios presentes. Revista Historia de la Educación Latinoamericana, Tunja, v. 11, p. 131-146, 2008.

DE SOUSA SANTOS, Boaventura. La universidad en el siglo XXI: para una reforma democrática y emancipatoria de la universidad. La Paz: CIDES-UMSA: ASDI: Plural, 2007.

DE SOUSA SANTOS, Boaventura. Constitución y hegemonía: luchas contra la dominación global. Chasqui, Quito, n. 136, p. 13-31, 2018.

DE SOUZA LIMA, Katia Regina. A política de ensino superior a distância no Brasil nos anos de neoliberalismo. Perspectiva, Florianópolis, v. 29, n. 1, p. 19-47, 2011.

DIAS SOBRINHO, José. Evaluación y reformas de la educación superior en América Latina. Perfiles Educativos, Ciudad de México, v. 27, n. 108, p. 31-44, 2005.

DIAS SOBRINHO, José. Avaliação e transformações da educação superior brasileira (1995-2009): do provão ao Sinaes. Avaliação, Campinas, v. 15, n. 1, p. 195-224, 2010.

DIAS SOBRINHO, José. Educação superior: bem público, equidade e democratização. Avaliação, Campinas, v. 18, n. 1, p. 107-126, 2013.

DÍAZ ESCOTO, Alma Silvia. La crisis de fin de siglo en la UNAM: financiamiento y gratuidad. Educação e Pesquisa, São Paulo, v. 33, n. 1, p. 81-94, 2007.

DIODATI, Mariano; MENDÍAZ, María Graciela. La retórica institucional del Banco Mundial en torno a las políticas de ciencia y tecnología de las universidades latinoamericanas. Hacia la Búsqueda de autonomía académica en los países de la región. Revista de la Escuela de Ciencias de la Educación, Rosario, v. 5, p. 51-68, 2010. 
DOURAD0, Luiz Fernandes. Reforma do Estado e as políticas para a educação superior no Brasil nos anos 90. Educação \& Sociedade, Campinas, v. 23, n. 80, p. 234-252, 2002.

DURÃO, Fabio Akcelrud. Las transformaciones en la concepción de universidad y el caso brasileño. Literatura, Bogotá, , v. 17, n. 2, p. 41-58, 2015.

EDELSTEIN, Gloria. ¿Qué docente hoy en y para las universidades? InterCambios, Montevideo, v. 1, p. 28-32, 2012.

ESCALANTE GONZALBO, Fernando. Historia mínima del neoliberalismo. México, DC: El Colegio de México, 2015.

ESPINOZA, Oscar. Neoliberalismo y educación superior en Chile: una mirada crítica al rol desempeñado por el Banco Mundial y los "Chicago Boys". Laplage em Revista, Sorocaba, v. 3, n. 3, p. 93-114, 2017.

FARAH, Ivonne. Presentación. In: DE SOUSA SANTOS, Boaventura (ed.). La universidad en el siglo XXI: para una reforma democrática y emancipatoria de la universidad. La Paz: CIDES-UMSA: ASDI: Plural, 2007. p. 7-10.

FASSBENDER, Dina Sophie; SILVA DÍAZ, John Alexander. ¿Pago por rendimiento? Una discusión de las políticas de incentivación de la productividad académica en Colombia y Alemania. Revista Papeles, Bogotá, v. 6, n. 11, p. 10-19, 2014.

FERNÁNDEZ JILBERTO, Alex E.; RIETHOF, Marieke. América Latina en el neoliberalismo global: crisis financiera, políticas sociales y reformas laborales. European Review of Latin American and Caribbean Studies, Amsterdam, n. 71, p. 125-137, 2001.

FERREIRA, Francisco H. G.; WALTON, Michael. La desigualdad en América Latina: ¿Rompiendo con la historia? Bogotá: Banco Mundial, 2005.

FIOL, Ana. Alca: una hegemonía dificilde lograr. Chasqui, Quito, n. 80, p. 17-23, 2002.

GALINDO, Camila Andrea; GÓMEZ, John Freddy; RODRÍGUEZ, María Alejandra. Repercusión del proyecto neoliberal en la educación superior en Colombia. Revista El Ágora USB, Medellín, v. 15, n. 1, p. 73-94, 2015.

GALLARDO CÓRDOVA, Katherina Edith et al. Evaluación de los estudiantes: las prácticas institucionales y docentes vistas desde el marco de estándares internacionales. Revista Apertura, Guadalajara, v. 7, n. 2, p. 73-85, 2015.

GARCÉS CORREA, Santiago et al. Trabajadores, recuperación de fábricas y neoliberalismo en Colombia (1995-2015). Revista Colombiana de Sociología, Bogotá, v. 40, n. 2, p. 239-256, 2017.

GARCÍA, Mía Dragnic; ORTIZ-CONTRERAS, Raúl. La mercantilización de la educación superior y la irrupción del movimiento estudiantil en Chile (2006/2011): impactos y desafíos. Temáticas, Campinas, v. 24, n. 47/48, p. 77-98, 2016. 
GAVIRIA RÍOS, Mario Alberto. Neoliberalismo y desarrollo en América Latina: una discusión sobre las reformas estructurales promovidas por el "Consenso de Washington". Páginas, Pereira, n. 69, p. 7-27, 2004.

GENTILI, Pablo. El Consenso de Washington y la crisis de la educación en América Latina. In: ÁLVAREZ-URÍA RICO, Fernando (ed.). Neoliberalismo versus democracia. Madrid: La Piqueta, 1998. p. 102-129.

GENTILI, Pablo. El (0)caso de la sociología de la educación en tiempos neoliberales: privatización del espacio público y reconversión intelectual. Revista de Educación, Madrid, n. 324, p. 49-60, 2001.

GÓMEZ-MORALES, Yuri Jack. El baile de los que sobran: cambio cultural y evaluación académica. Revista Colombiana de Antropología, Bogotá, v. 53, n. 2, p. 15-25, 2017.

GONZÁLEZ LUNA, Fernando. Breve ensayo sobre los escenarios de las universidades del siglo XXI: notas para la reflexión y diálogo en la nueva fase neoliberal. Visión Educativa lunaes, Durango, v. 11, n. 23, p. 100-105, 2017.

HARVEY, David. Breve historia del neoliberalismo. Madrid: Akal, 2007.

HERNÁNDEZ GUTIÉRREZ, Rubén. Trayectoria del neoliberalismo: de la academia al espacio público. Revista Mexicana de Ciencias Políticas y Sociales, Ciudad de México, v. 61, n. 227, p. 423-428, 2016.

HEYWOOD, Andrew. Political ideologies: an introduction. 6. ed. Basingstoke: Palgrave Macmillan, 2017.

HEYWO0D, Andrew. Politics. 4. ed. Basingstoke: Palgrave Macmillan, 2013.

IBARRA, David. 0 neoliberalismo na america Latina. Revista de Economia Política, Madrid,v. 31, n. 2, p. 238-248, 2011.

JIMÉNEZ ORTIZ, María del Carmen. El discurso mundial de modernización educativa: evaluación de la calidad y reforma de las universidades latinoamericanas. Espacio Abierto Cuaderno Venezolano de Sociología, Maracaibo, v. 20, n. 2, p. 219-238, 2011.

JULIÁN VEJAR, Dasten. Precariedad laboral en América Latina: contribuciones a un modelo para armar. Revista Colombiana de Sociología, Bogotá, v. 40, n. 2, p. 27-46, 2017.

KATZ, Claudio. Los atolladeros de la economía latinoamericana. Serviço Social \& Sociedade, São Paulo, n. 112, p. 711-728, 2012.

LAMPERT, Ernani. El declive de la universidad pública: la interface de una ideología. Perfiles Educativos, Ciudad de México, v. 27, n. 109-110, p. 84-116, 2005.

LARA, José Bell; LÓPEZ, Delia Luisa. La cosecha del neoliberalismo en América Latina. Semantic Scholar, p. 1-25, 2002. Disponible en: http://www.insumisos.com/lecturasinsumisas/Neoliberalismo en America Latina.pdf. Acceso en: 18 dic. 2019. 
LEAL VILLEGAS, Paulo Humberto. Latinoamérica ante las transformaciones del orden monetario y financiero mundial. Economía Informa, Ciudad de México, v. 396, p. 67-83, 2016.

LEITE, Denise; GENRO, Maria Elly Herz. Avaliação e internacionalização da educação superior: Quo vadis América Latina? Avaliação, Campinas, v. 17, n. 3, p. 763-785, 2012.

LEVINE, Barry B. (ed.). El desafío neoliberal: el fin del tercermundismo en América Latina. Bogotá: Norma, 1992.

LIMA, Licínio C.; AZEVED0, Mário Luiz Neves de; CATANI, Afrânio Mendes. 0 processo de Bolonha, a avaliação da educação superior e algumas considerações sobre a Universidade Nova. Avaliação, Campinas, v. 13, n. 1, p. 7-36, 2008.

LONDOÑO OROZCO, Guillermo. La docencia universitaria: realidad compleja y en construcción. Miradas desde el estado del arte. Itinerario Educativo, Cali, v. 29, n. 66, p. 47-85, 2015.

LÓPEZ CASTELLANOS, Nayar. América Latina: recomposición hegemónica y resistencia emancipatoria. Pacarina del Sur, En línea, v. 27, 2016. Disponible en: http://pacarinadelsur.com/home/abordajes-ycontiendas/1283-america-latina-recomposicion-hegemonica-y-resistencia-emancipatoria. Acceso en: 29 nov. 2019.

LÓPEZ GUERRA, Susana; FLORES CHÁVEZ, Marcelo. Las reformas educativas neoliberales en Latinoamérica. Revista Electrónica de Investigación Educativa, Ensenada, v. 8, n. 1, p. 1-15, 2006.

LÓPEZ MERAZ, Óscar Fernando; VILLEGAS LOEZA, Diana. Hegemonía selectiva en América Latina: puntos neoliberales de encuentro entre México y Colombia. Espacio Abierto Cuaderno Venezolano de Sociología, Maracaibo, v. 24, n. 2, p. 207-222, 2015.

LORUSSO, Fabrizio. Management y privatismo: pilares ideológicos del neoliberalismo y la americanización en América Latina. Política y Cultura, Ciudad de México, n. 43, p. 95-123, 2015.

MACEIRA OCHOA, Luz María. El sueño y la práctica de sí: pedagogía feminista: una propuesta. Ciudad de México: El Colegio de México: Centro de Estudios Sociológicos: Programa Interdisciplinario de Estudios de la Mujer, 2008.

MACEIRA OCHOA, Luz María. Más allá de la coeducación: pedagogía feminista. Educar, Guadalajara, n. 36, p. 27-36, 2006.

MAIA, João Marcelo. Ciências sociais, trabalho intelectual e autonomia: quatro estudos de caso sobre nós mesmos. Dados, Rio de Janeiro, v. 62, n. 2, p. 1-33, 2019.

MAÑUNGA ARROYO, Ángela María. Un paso adelante, dos atrás: lógicas de mercado y políticas de exclusión en Colombia. Trans-pasando Fronteras, Cali, n. 8, p. 19-35, 2015.

MARTÍNEZ ALCÁNTARA, Susana; PRECIADO SERRANO, María de Lourdes. Consecuencias de las políticas neoliberales sobre el trabajo y la salud de académicos universitarios: el burnout como fenómeno emergente.

Psicología y Salud, Xalapa, v. 20, n. 1, p. 119-128, 2010. 
MARTíNEZ B00M, Alberto. Malestar docente y profesionalización en América Lalina. Revista Española de Educación Comparada, Madrid, n. 6, p. 87-112, 2000.

MASSÉ NARVÁEZ, Carlos Eduardo. Autonomía estatal y universitaria, mercantilización del conocimiento y educación en el neoliberalismo. Educere, Mérida, v. 12, n. 41, p. 387-395, 2008.

MELO, Adriana Almeida Sales de. A mundialização da educação: o projeto neoliberal de sociedade e de educação no Brasil e na Venezuela. Trabalho, Educação e Saúde, Rio de Janeiro, v. 3, n. 2, p. 397-408, 2005.

MÉNDEZ REYES, Johan; MORÁN BELTRÁN, Lino. La universidad en tiempos de incertidumbre. Opción, Maracaibo, v. 28, n. 68, p. 393-407, 2012.

MIÑANA BLASCO, Carlos; RODRÍGUEZ, José Gregorio. La educación en el contexto neoliberal. In: RESTREPO BOTERO, Dario Indalecio (ed.). La falacia neoliberal: crítica y alternativas. Bogotá: Universidad Nacional de Colombia, 2003. p. 285-321.

MORA CORTÉS, Andrés Felipe. Veinticinco años de crisis fiscal en Colombia (1990-2014): acumulación, confianza y legitimidad en el orden neoliberal. Papel Político, Bogotá, v. 20, n. 1, p. 63-99, 2015.

MORA MORAGA, Flavio; GUTIÉRREZ ESPELETA, Ana Lucía. La acción social en la universidad pública: actuando en la era neoliberal. Revista de Ciencias Sociales, San José, n. 148, p. 49-66, 2015.

MORALES HERRERA, Milton Danilo. Prácticas de resistencia docente en la universidad orientada al mercado: capturas y fugas académicas en el contexto colombiano. Barcelona: UAB, 2012. 357 p. Tesis (Doctorado) - Departamento de Psicología Social, Universidad Autónoma de Barcelona, Barcelona, 2012.

NARODOWSKI, Mariano. Cuasimonopolios escolares: lo que el viento nunca se llevó. Revista Educación y Pedagogía, Medellín, v. 22, n. 58, p. 29-36, 2010.

NAVARRETE SAAVEDRA, Rodrigo. Gobernabilidad neoliberal y movimientos indígenas en América Latina. Polis, Santiago, n. 27, p. 1-17, 2010.

NETTO MACHADO, Ana María. Universidades comunitarias: un modelo brasileño para interiorizar la educacion superior. Universidades, Ciudad de México, n. 37, p. 37-48, 2008.

ORNELAS DELGAD0, Jaime. Neoliberalismo y capitalismo académico. In: GENTILI, Pablo et al. (ed.). Políticas de privatización, espacio público y educación en América Latina. Rosario: Homosapiens, Clacso, 2009. p. 83-119.

PÉREZ, Marta; MONTOYA, Ainhoa. La insostenibilidad de la universidad pública neoliberal: hacia una etnografía de la precariedad en la Academia. Revista de Dialectología y Tradiciones Populares, Madrid, v. 73, n. 1, p. 9, 2018.

PÉREZ-BUSTOS, Tania. Mi tiempo ya no es mío: reflexiones encarnadas sobre la cienciometría. Nómadas, Bogotá, n. 50, p. 35-43, 2019. 
PÉREZ-BUSTOS, Tania; BOTERO-MARULANDA, Daniela. Otras prácticas educomunicativas: otras sexualidades, potencialmente otro ethos científico. Revista de Estudios Sociales, Bogotá, n. 49, p. 113127, 2014.

PIRES, Marília Freitas de Campos; REIS, José Roberto Tozoni. Globalização, neoliberalismo e universidade: algumas considerações. Interface - Comunicação, Saúde, Educação, Botucatu, v. 3, n. 4, p. 29-39, 1999.

PORTES, Alejandro; HOFFMAN, Kelly. La estructura de clases en América Latina: composición y cambios durante la era neoliberal. Desarrollo Económico, Santiago, v. 43, n. 71, p. 355-387, 2003.

RAMÍREZ, Hernán. Las dictaduras del Cono Sur y el giro de les políticas económicas. Boletín Americanista, Barcelona, n. 72, p. 199-220, 2016.

RAMOS RUIZ, Yolanda; PARRA RAMÍREZ, Agustin. Programa "Ser pilo paga" en el marco de la educación superior en Colombia. Diálogos sobre Educación, Guadalajara, v. 14, n. 8, p. 1-18, 2017.

RAZA, Werner G. Desarrollo capitalista, neoliberalismo y ambiente en América Latina: una breve sinopsis. Ecología Política, Barcelona, n. 20, p. 153-165, 2000.

RIBEIR0, Maria das Graças M. Políticas para a educação superior no Cone Sul: do autoritarismo ao neoliberalismo. Pro-Posições, Campinas, v. 11, n. 3, p. 55-65, 2000.

RIVERO BOTTERO, Raquel. Educación y pedagogía en el marco del neoliberalismo y la globalización. Perfiles Educativos, Ciudad de México, v. 35, n. 142, p. 149-166, 2013.

RODRIGUEZ PECIAR, Paola Luciana. A educação superior universitária frente aos desafios das mudanças do mundo do trabalho no contexto do neoliberalismo. Reflexão e Ação, Santa Cruz do Sul, v. 17, n. 2, p. 09-25, 2009.

SANTANA CASTILLO, Joaquín. Neoliberalismo, gobernabilidad y nuevos movimientos sociales. El cambio político en diferentes naciones de Latinoamérica. CLI0 América, Santa Marta, v. 2, n. 4, p. 213-237, 2014.

SANTARRONE, Fernanda; VITTOR, Ariel. La neo educación liberal: una visión general acerca de las ideas neoliberales sobre la educación. Aula Abierta, Oviedo, v. 32, n. 83, p. 3-20, 2004.

SANTOS, Vivian Matias. Políticas públicas em educação: a "lógica subalternizante" vigente na América Latina e seus reflexos na universidade brasileira (Educational policy : subaltern politic Latin American in the Brazilian university). Emancipação, Ponta Grossa, v. 8, n. 1, p. 79-93, 2008.

SERAFIM, Milena Pavan. 0 processo de mercantilização das instituições de educação superior: um panorama do debate nos EUA, na Europa e na América Latina. Avaliação, Campinas, v. 16, n. 2, p. 241-265, 2011.

SGUISSARDI, Valdemar; SILVA JR., João dos Reis. La forma y los motivos de la expansión de la educación superior pública en Brasil. Revista de la Educación Superior, Ciudad de México, v. 41, n. 162, p. 67-86, 2012. 
SILVA MONTES, César. Neoliberalismo y educación superior en el norte de México. Revista Educación y Humanismo, Barranquilla, v. 13, n. 21, p. 112-128, 2011.

SOARES CAMPOS, Rosana. 0 impacto das reformas econômicas neoliberais na América Latina: desemprego e pobreza. Polis, Santiago, v. 47, n., p. 1-20, 2017.

STROMQUIST, Nelly P. Educação Latino-Americana em tempos globalizado. Sociologias, Porto Alegre, v. 14, n. 29, p. 72-99, 2012.

TEODORO, Antonio; GUILHERMINA, Manuela. A educação superior em tempos de mudança na América Latina e na Europa: anotações para uma agenda alternativa. Laplage em Revista, Sorocaba, v. 3, n. 3, p. 8, 2017.

THOMPSON, Julie. Educación superior y ajuste estructural en Costa Rica. Perfiles Educativos, Ciudad de México, v. 70, p. 3-14, 1995.

TORRES, Carlos Alberto; SCHUGURENSKY, Daniel. La economía política de la educación superior en la era de la globalización neoliberal: América Latina desde una perspectiva comparatista. Perfiles Educativos, Ciudad de México, v. 23, n. 92, p. 6-31, 2001.

TORRES GONZÁLES, Jaime. La influencia de la política neoliberal en la sociedad Colombiana y el fortalecimiento del autoritarismo. 2009. 461 p. Tesis (Doctorado) - Fachbereich Politik- und Sozialwissenschaften, Otto Suhr Institut für Politikwissenschaft, Freie Universität Berlin, Berlín, 2009.

VEGA CANTOR, Renan. Contrarreforma educativa en Colombia. El Ágora USB, Medellín, v. 11, n. 2, p. 335-379, 2011.

VILAS, Carlos M. Las "idas" y "regresos" del Estado. Utopía y Praxis Latinoamericana, Maracaibo, v. 15, n. 49, p. 101-108, 2010.

VILLAMIZAR MARULANDA, Édgar; URIBE ARBELÁEZ, Martín. El fracaso del neoliberalismo y su modelo de desarrollo. Revista La Propiedad Inmaterial, Bogotá, n. 13, p. 119-150, 2009.

VIZCAíNO G., Milcíades. Selección/exclusión: dilema de las universidades en América Latina. Pedagogía y Saberes, Bogotá, n. 23, p. 47-63, 2005.

ZAPATA, Gonzalo; TEJEDA, Ivo. Impactos del aseguramiento de la calidad y acreditación de la educación superior. Consideraciones y proposiciones. Calidad en la Educación, Providencia, n. 31 , p. 192-209, 2009.

ZAPATA-SEPÚlVEDA, Pamela; JIMÉNEZ-BENITEZ, Víctor; CONCHA-ASTORGA, David. From the struggle for education to the transformation of society. Cultural Studies Critical Methodologies, Londres, v. 13, n. 6, p. 497-503, 2013. 
Recibido en: 10.01.2020

Revisado en: 30.06.2020

Aprobado en: 01.09.2020

Isaura Castelao-Huerta es candidata a doctora en Ciencias Humanas y Sociales por la Universidad Nacional de Colombia, sede Bogotá, en donde hace parte del Grupo Interdisciplinario de Estudios de Género. 